\title{
Lung function in primary Sjögren's syndrome: a cross sectional and longitudinal study
}

\author{
Clive Kelly, Phillip Gardiner, Badal Pal, Ian Griffiths
}

\begin{abstract}
Clinical and radiological assessment of 100 patients (97 female) with primary Sjögren's syndrome was performed within six months of diagnosis in conjunction with spirometry and measurement of transfer factor for carbon monoxide (TLCO). This was repeated in an unselected subgroup of 30 patients after a mean interval of four years. On initial assessment, 43 patients had symptoms of lung disease and 10 had related physical signs; the chest radiograph was abnormal in five. There was a significant reduction (more than 2 standardised residuals) in forced expiratory volume in one second $\left(F E V_{1}\right)$, vital capacity (VC), and TLCO in 14, 12, and 10 patients, 24 patients overall having a significant reduction in one or more of these measures. There was a strong relation between reduction in lung function and both pulmonary symptoms and a lip biopsy specimen positive for Sjögren's syndrome. Lung function at the initial assessment in the 30 patients who were restudied was almost identical to that of the group as a whole. Seventeen now had symptoms and nine had related physical signs. The chest radiograph was abnormal in four patients. More patients had a significant reduction in $F E V_{1}, V C$, and TLCo. Lung disease is sometimes an early feature of primary Sjögren's syndrome and may progress over a relatively short period.
\end{abstract}

Lung function has been studied in several cross sectional series of patients with primary Sjögren's syndrome. ${ }^{1-8}$ Interstitial lung disease and small airways obstruction have been found frequently and may coexist in some patients. ${ }^{1-6}$ Less commonly, large airways obstruction and pleural disease have been recorded. ${ }^{4}$ Several of these studies, however, have been complicated by the inclusion of patients with both primary and secondary Sjögren's syndrome $e^{1-36-8}$ or by failure to describe their smoking habits. ${ }^{13}$ Furthermore, no longitudinal studies have been performed and the relation between pulmonary function and symptoms of chest disease has not been investigated.

We report the results of simple lung function tests performed in 100 patients with primary Sjögren's syndrome and relate the findings to their symptoms and signs and to radiological and serological markers of disease activity. The assessment was repeated after a mean of four years in an unselected group of 30 patients.

\section{Methods}

\section{RECRUITMENT OF PATIENTS}

Patients referred to a special clinic for evaluation of possible primary Sjögren's syndrome had a series of investigations, including (a) Schirmer's tests and Rose Bengal staining of the conjunctiva; (b) serological tests for antinuclear factor and IgM rheumatoid factor; $(c)$ salivary flow estimation; and $(d)$ biopsy of the lip salivary gland. Serum was also sent to the Kennedy Institute at the Royal Free Hospital in London for measurement of anti-Ro and anti-La antibodies in all patients. ${ }^{9}$ We used the objective criteria for the diagnosis of primary Sjögren's syndrome proposed by Fox et $a l,{ }^{10}$ with the single modification that, if formal parotid duct cannulation could not be achieved, subjective xerostomia supported by the absence of a pool of saliva under the tongue on examination was accepted as a criterion (table 1). The diagnosis of primary Sjögren's syndrome was considered to be "definite" in those patients who fulfilled all four diagnostic criteria and "probable" in patients fulfilling only three criteria. None of our patients fulfilled standard American Rheumatology Association criteria for a related connective tissue disease.

\section{ASSESSMENT}

In 100 consecutive patients with definite or probable primary Sjögren's syndrome symptoms and signs of possible pulmonary disease were sought and recorded, together with a smoking history. A chest radiograph was performed within six months of referral after the following simple assessments of lung function had been completed: forced expiratory volume in one second $\left(\mathrm{FEV}_{1}\right)$, relaxed vital capacity (VC) and carbon monoxide transfer factor (TLCO) and transfer coefficient (KCO). Results were expressed as standardised residuals (SR). ${ }^{11}$ Change in $\mathrm{FEV}_{1} 30$ minutes after an inhaled dose of $400 \mu \mathrm{g}$ of salbutamol was recorded in patients with an $\mathrm{FEV}_{1}$ reduced by at least $10 \%$ from predicted. All patients were seen at least six monthly for review.

\section{REVIEW}

In 30 of the earliest patients to be fully documented in the clinic the assessment of symptoms and signs of possible chest disease was repeated after a mean interval of four years. Any change in smoking habit was 
Table 1 Diagnostic criteria used for primary Sjögren's syndrome (at least three necessary for diagnosis) *

1 Symptomatic xerostomia

2 Abnormal response to Schirmer's test ( $<10 \mathrm{~mm}$ wetting in $5 \mathrm{~min}$ bilaterally)

3 Positive result in serological tests (antinuclear factor or rheumatoid factor $>1 / 40$ ) or "+" anti-Ro antibody

4 Positive lip biopsy specimen (grades II, III, or IV)

*Exclusions include any patients with pre-existent rheumatoid arthritis, systemic lupus erythematosus, sarcoidosis, or lymphoma.

recorded. Chest radiography and lung function tests were then repeated. Investigations were repeated in the other 70 patients only when they were thought to be clinically indicated.

\section{STATISTICAL ANALYSIS}

The method of standardised residuals (SR) was used to assess baseline pulmonary function and to express change with time. Analysis of variance was used to assess the relation between lung function and other variables (the presence or absence of lip biopsy specimens positive for Sjögren's syndrome, anti-Ro antibody, pulmonary symptoms, and smoking). The two sample $t$ test was used to compare baseline values between the group of patients who were followed and those who were not. The paired $t$ test was used to assess change in lung function with time.

\section{Results}

The mean age at diagnosis of our 100 patients was 48 (range 15-75) years, and 97 were female. The results of the diagnostic investigations are summarised in table 2 , and described in detail elsewhere. ${ }^{12}$ Anti-Ro antibodies were detected in 31 patients.

\section{BASELINE FINDINGS \\ Clinical}

At diagnosis 16 patients were current smokers, and 12 were ex-smokers. Respiratory symptoms were present in 43 patients, predominantly dyspnoea and cough. Three patients had also had intermittent pleuritic chest pain associated with dyspnoea and in one case with wheeze. Signs of chest disease were present in 10 patients, with bilateral fine basal lung crackles noted in six and wheeze in two. One patient had signs of bilateral small pleural

Table 2 Results of diagnostic investigations in 41 patients with definite and 59 patients with probable primary Sjögren's syndrome

\begin{tabular}{lrrrr}
\hline Investigation & $L B$ & $S T$ & $S e$ & $X e$ \\
\hline $\begin{array}{l}\text { Definite primary Sjögren's syndrome } \\
\quad(4 \text { criteria) }\end{array}$ & 41 & 41 & 41 & 41 \\
Probable primary Sjögren's syndrome & & & & \\
$\quad(3$ criteria) & 10 & 10 & 0 & 10 \\
LB +, ST+, Xe+ & 13 & 0 & 13 & 13 \\
LB+, Se+, Xe+ & 0 & 36 & 36 & 36 \\
ST +, Xe+, Se+ & 64 & 87 & 90 & 100 \\
Total No with positive results & & & &
\end{tabular}

LB-Lip biopsy of minor salivary glands ( $>1$ focus $/ 4 \mathrm{~mm}^{2}$ ); ST-Schirmer's test ( $<9 \mathrm{~mm}$ wetting each eye); $\mathrm{Xe}-$ reduced salivary flow rate $(<1 \mathrm{ml} / \mathrm{min}$ ) or xerostomia; Se-positive serological result (antinuclear factor or rheumatoid factor $>1 / 40$ or " +" anti-Ro antibody). effusions and another of stridor. No patient had finger clubbing or central cyanosis.

\section{Radiological}

Chest radiographs were abnormal in five patients, with alveolitis present in three cases. Bilateral pleural effusions and a pericardial effusion were detected in one patient with normal cardiac function, and one individual with stridor had bilateral paratracheal lymphadenopathy with exophthalmos but no histological evidence of lymphoma. The latter is the subject of a separate report. ${ }^{13}$

\section{Physiological}

Figure 1 shows the baseline lung function of all patients, using standardised residuals. Gas transfer measurements could not be obtained in four patients owing to low lung volumes. The reductions in $\mathrm{FEV}_{1}, \mathrm{VC}$, and TLCo were significant (over 2 SR) in 14,12, and 10 patients respectively. In total, 24 individuals had a significant reduction in one or more of these measurements on initial assessment. The mean $\mathrm{FEV}_{1} / \mathrm{VC}$ ratio was $77 \%$ and was less than $60 \%$ in five patients, exceeding $90 \%$ in only one. The change in $\mathrm{FEV}_{1}$ that followed inhalation of salbutamol was measured in 36 patients and an improvement in $\mathrm{FEV}_{1}$ of more than $15 \%$ occurred in four patients $(11 \%)$.

There was a strong relation between the presence of pulmonary symptoms and a reduction in $\mathrm{FEV}_{1}$ and VC ( $\left.<<0.001\right)$; symptoms were related less closely to low transfer factor $(p<0.05)$. Patients with a lip biopsy specimen positive for Sjögren's syndrome had a lower $\mathrm{FEV}_{1}$ and $\mathrm{VC}(\mathrm{p}<0.01)$. The presence of anti-Ro antibody was related to these measures less closely $(p<0.05)$ We did not detect a significant influence of smoking on lung function, possibly because of the relatively few smokers.

\section{FINDINGS AT REVIEW}

The 30 patients who had lung function tests repeated after a mean of four years had a median age of 53 years at diagnosis and included only one man. No differences were found between this group and the other 70 patients at the initial examination with respect to pulmonary symptoms, smoking, lip biopsy positivity, anti-Ro antibody, or baseline lung function.

\section{Clinical and radiological}

Two patients had stopped smoking during the follow up period, leaving three current smokers in this group. Four patients developed dyspnoea during follow up, so that 17 patients now reported pulmonary symptoms. Physical signs of chest disease had been detected initially in four patients but were noted in nine patients at review. Bilateral fine crackles were present in all nine, and one had features of a pleural effusion. The chest radiograph, initially abnormal in two patients, had became abnormal in a further two during the follow up period. All showed evidence of alveolitis, with associated persistent pleural effusions in one patient. 
Standardised residual

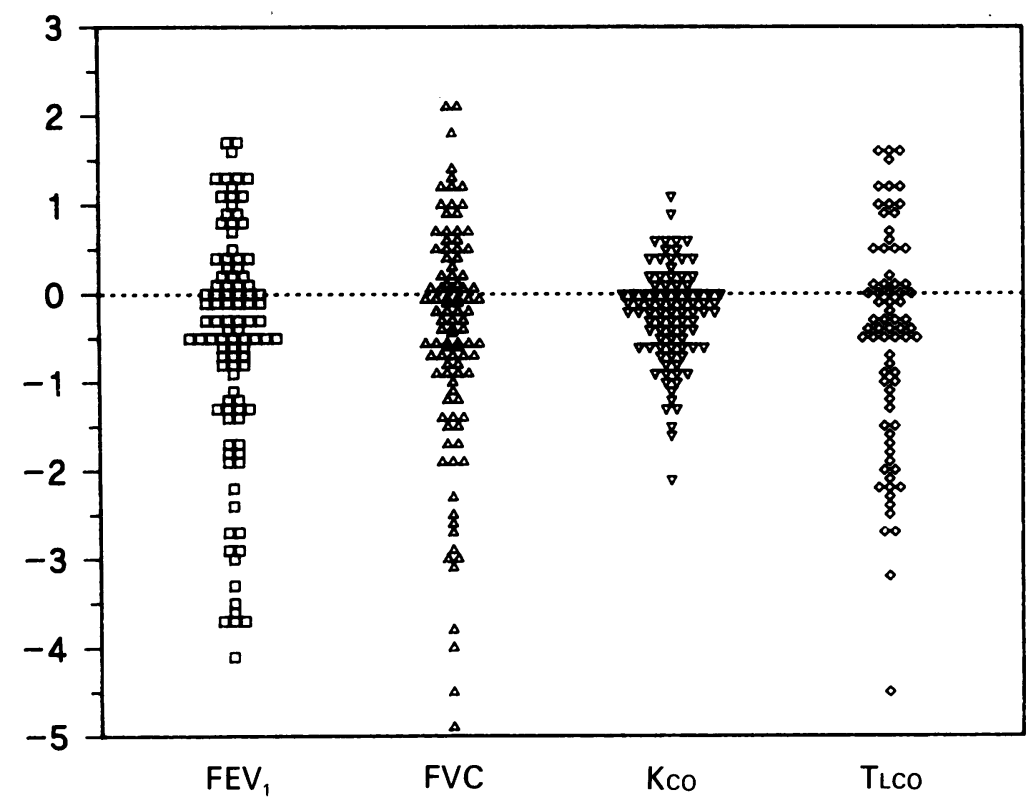

Figure 1 Baseline pulmonary function-forced expiratory volume in one second $\left(F E V_{1}\right)$, vital capacity (VC), carbon monoxide transfer factor (TLCO), and transfer coefficient (KCO) -in 100 patients at presentation with primary Sjögren's syndrome expressed in terms of standardised residuals.
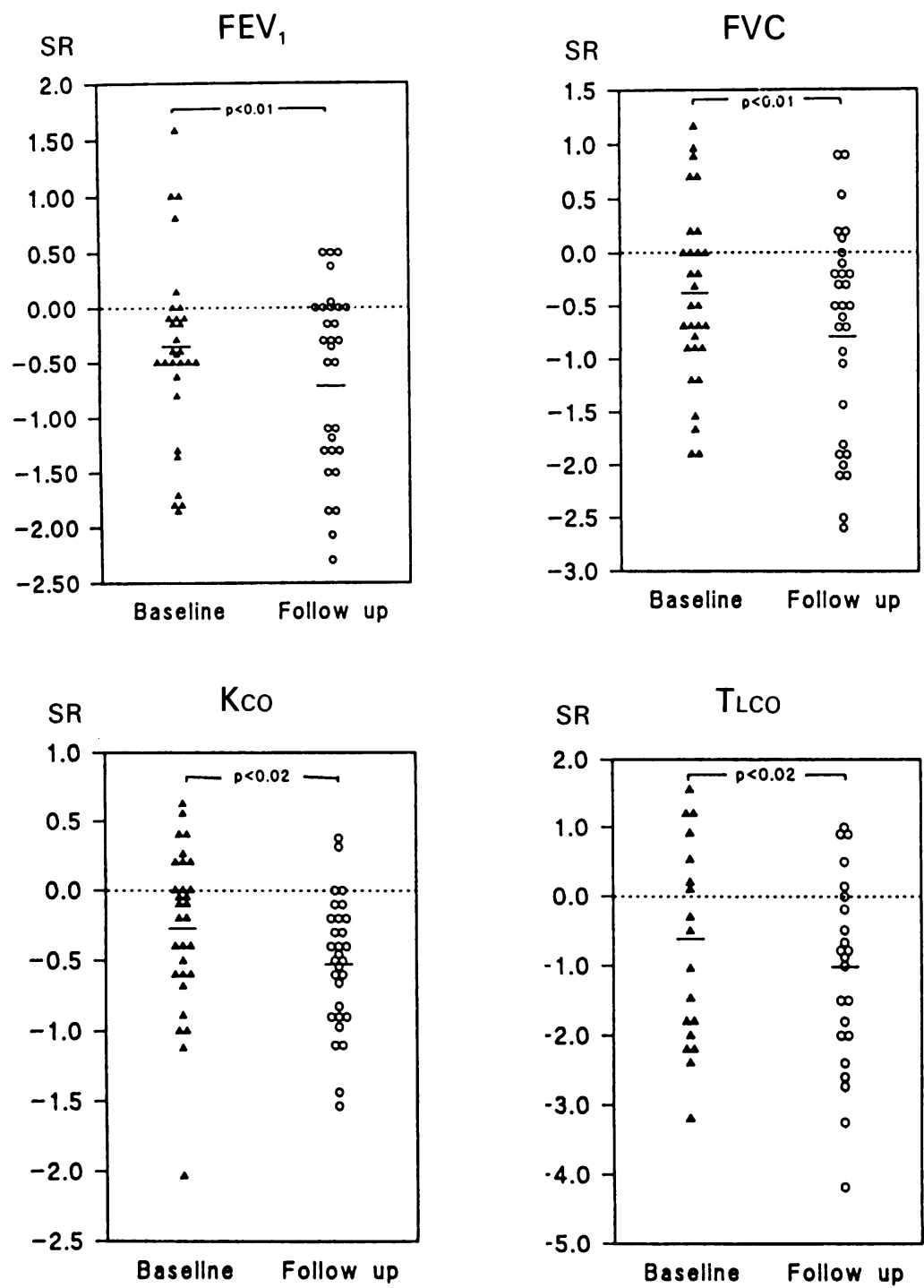

Figure 2 Changes in standardised residual (SR) FEV, VC, TLCO, and Kco over a mean follow up of four years in 30 patients with primary Sjögren's syndrome. The mean is represented by a horizontal bar in each figure and significance is calculated by paired $t$ test. Abbreviations as in figure 1.

\section{Physiological}

Figure 2 summarises the changes in pulmonary function in these 30 patients. There was a significant reduction in all measurements when the effects of age, height, and sex were taken into account. The mean annual reduction in FEV was $35 \mathrm{ml}$.

Of the other 70 patients who remained under review but did not routinely undergo repeat investigation, one died from an adenocarcinoma of the lung and another required cytotoxic drugs for upper airway obstruction. ${ }^{13}$ None of our patients has developed pulmonary lymphoma or pulmonary vasculitis during follow up.

\section{Discussion}

Over $40 \%$ of our patients had pulmonary symptoms at presentation, though physical signs and radiographic abnormalities were rarely detected at this stage. Almost one quarter of our patients had a significant reduction in lung function on initial assessment and these invariably had symptoms.

Several specific pulmonary complications have been reported in patients with primary Sjögren's syndrome, including pulmonary lymphoma and pseudolymphoma, ${ }^{14}$ atelectasis and bronchiectasis, ${ }^{38}$ pulmonary hypertension, ${ }^{15}$ and pulmonary vasculitis. ${ }^{16} \mathrm{We}$ did not identify any of these problems in our 100 patients and, although a few individuals developed infective bronchitis, this was a recurrent problem in only one patient. ${ }^{13}$ None the less, we did observe a high incidence of pulmonary symptoms and this is in keeping with many previous reports. ${ }^{1-8}$ In our series dyspnoea and cough were more frequent than either wheeze or chest pain, suggesting that the lesions did not usually lie within the major airways or the pleural spaces.

Whether an obstructive or a restrictive pattern of abnormality predominates according to pulmonary function tests is debated. ${ }^{1-6}$ Our investigations were insufficiently sensitive to detect minor degrees of small airways obstruction, but we did not find a high incidence of airways obstruction of clinical relevance. There was also little reversibility of $\mathrm{FEV}_{1}$ in response to a bronchodilator, an increase in $\mathrm{FEV}_{1}$ of more than $15 \%$ being seen in only $11 \%$ of those tested. Severe restriction was even less common in our series as only one patient had an $\mathrm{FEV}_{1} / \mathrm{VC}$ ratio greater than $90 \%$. We do not find the traditional division of lung disease in primary Sjögren's syndrome into obstructive and restrictive patterns to be helpful, and suggest that classification should be based on histological findings alone.

The patients with a lip biopsy specimen positive for Sjögren's syndrome had a sig-

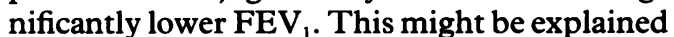
by more advanced disease in these individuals or by a greater propensity for systemic disease, as manifested by the increased frequency of anti-Ro antibody. ${ }^{17}$ Evidence of histological abnormality in the lungs of patients with primary Sjögren's syndrome comes almost exclusively from case reports. Newball and Bramin described two patients with infiltration of the small airways by lymphocytes and inflam- 
matory cells causing oedema and luminal narrowing. ${ }^{2}$ Although lymphocytic interstitial pneumonitis is a recognised complication of primary Sjögren's syndrome it is rare, the histological features being described in a 1977 review. ${ }^{18} \mathrm{~A}$ study of symptomless patients with normal pulmonary function using bronchoalveolar lavage indicated a high incidence of lymphocytosis. ${ }^{19}$ Although the meaning of these findings is uncertain without follow up data, they raise the possibility that the pulmonary features of primary Sjögren's syndrome are due at least in part to activated lymphocytes. Further support for this theory may be gleaned from the fact that lymphoma is a well recognised complication of primary Sjögren's syndrome $\mathrm{e}^{20}$ and that a broad range of lymphocytic transformation, from benign to frankly malignant, may occur. ${ }^{21} \mathrm{~A}$ recent report suggests that $20 \%$ of patients with Sjögren's syndrome who have lymphoma have evidence of affected lungs, and that pulmonary lymphoma may present with features clinically indistinguishable from those of lymphocytic interstitial pneumonitis. ${ }^{22}$

At review four years after the initial assessment chest symptoms, signs, and radiographic abnormalities had all increased in incidence. Standardised residuals obtained from cross sectional data were used to look at the change with time because there are insufficient data from longitudinal surveys. Significant falls in all lung function measurements had occurred after correction for age and several patients had now developed dyspnoea, crackles, and interstitial shadowing.

Smoking appears to be less common than expected in patients with established primary Sjögren's syndrome, possibly because sicca symptoms are exacerbated by cigarette smoke. Although lung damage due to smoking may accelerate the pulmonary features of primary Sjögren's syndrome it does not explain our findings, which appear to be related more closely to markers of severe or systemic disease. ${ }^{23}$

In this relatively young population with a low incidence of smoking our findings suggest that pulmonary complications of primary Sjögren's syndrome represent a significant threat to health and account for considerable morbidity. The pathophysiological mechanisms leading to pulmonary disease in primary Sjögren's syndrome await elucidation.

We wish to thank Dr Alastair Brewis for his critical appraisal of our work, and Mr Christopher Ward and Mrs Alison Avery for our work, and Mr Christopher Ward and Mrs Alison Avery for
help with the statistical analysis. We acknowledge the assistance of the nursing staff of the rheumatology department and of the pulmonary function technicians at the Royal Victoria Infirmary, together with the help provided by the staff of the immunology department of Newcastle General Hospital.

1 Segal I, Fink G, Machtey I, Gura V, Spitzer S. Pulmonary function abnormalities in Sjögren's syndrome and the sicca complex. Thorax 1981;36:286-9.

2 Newball HH, Brahim SA. Chronic obstructive airway disease in patients with Sjögren's syndrome. Am Rev Respir Dis 1977;115:295-303.

3 Strimlan CV, Rosenow EC, Divertie MB, Harrison EG. Pulmonary manifestations of Sjögren's syndrome. Chest 1976;70:354-61.

4 Constantopoulos SH, Drosos AA, Maddison PJ, Moutsopoulos HM. Xerotrachea and interstitial lung disease in primary Sjögren's syndrome. Respiration 1984;46:310-4.

5 Oxholm P, Bundgaard A, Birk Madsen E, Mathorpe R, Veljo Rasmussen F. Pulmonary function abnormalities in patients with primary Sjögren's syndrome. Rheumatol Int 1982;2:179-81.

6 Vitalli C, Tavoni A, Viegi G, Begliomini E, Agnesi A, Bombardieri S. Lung involvment in Sjögren's syndrome: a comparison between patients with primary and with secondary syndrome. Ann Rheum Dis 1985;44:455-61.

7 Ellman P, Weber WP, Goodier TEW. A contribution to the pathology of Siögren's syndrome. $Q J$ Med 1951;20: 33-42.

8 Fairfax AJ, Haslam PL, Pavia D, et al. Pulmonary disorders associated with Sjögren's syndrome. $Q$ J Med 1981; 50:179-95.

9 Venables PJW, Charles PJ, Buchanan RRC, et al. Quantitation and detection of isotypes of anti-SS-B antibodies by ELISA and Farr assays using affinity purified antigens. Arthr Rheum 1983;26:146-55.

10 Fox RI, Robinson C, Curd J, Cozin F, Howell FV. Sjögren's syndrome: proposed criteria for classification. Arthr Rheum 1986;29:577-85.

11 Miller MR, Pincock AC. Predicted values: how should we use them? Thorax 1988;43:265-8.

12 Kelly CA, Pal B, Gardiner P, et al. Primary Sjögren's syndrome in North East England-a longitudinal study. $B r J$ Rheum (in press).

13 Kelly CA, Griffiths ID. Major upper airways obstruction associated with Sjögren's syndrome: a case report and literature review. $B r J$ Rheum 1989;28:543-5.

14 Kamholz S, Sher A, Barland P, Rosen N, Rakoff S, Becker N. Sjögren's syndrome: severe upper airways obstruction due to primary malignant tracheal lymphoma developing during successful treatment of lymphocytic interstitial pneumonitis. $J$ Rheumatol 1987;143:588-94.

15 Hedgpeth MD, Boulware DW. Pulmonary hypertension in primary Sjögren's syndrome. Ann Rheum Dis 1988; 47:251-3.

16 Bucher UG, Reid L. Sjögren's syndrome: report of a fatal case with pulmonary and renal lesions. $\mathrm{Br} J$ Dis Chest 1959;53:237-52.

17 Greenspan JS, Daniels TE, Talal N, et al. The histopathology of Sjögren's syndrome in labial salivary gland biopsies. Oral Surg 1974;37:217-29.

18 Strimlan CV, Rosenow EC III, Weiland LH, Brown LR Lymphocytic interstitial pneumonitis: review of 13 cases. Ann Intern Med 1978;88:616-21.

19 Hatron P-Y, Wallaert B, Gosset D, et al. Subclinical lung inflammation in primary Sjögren's syndrome. Arthr Rheum 1987;30:1226-30.

20 Talal N, Bunim J. The development of malignant lymphoma in the course of Sjögren's syndrome. Am J Med 1964;36:529-33.

21 Anderson LG, Talal N. The spectrum of benign to malignant lymphoproliferation in Sjögren's syndrome. Clin Exp Immunol 1971;9:199-221.

22 Hansen LA, Prakash UB, Colby TV. Pulmonary lymphoma in Sjögren's syndrome. Mayo Clin Proc 1989;64:920-31.

23 Hansen B, Manthorpe R. Antibodies against SS-B/La and SS-A/Ro antigens in patients with primary Sjögren's syndrome. Scand J Rheumatol 1986;suppl 61:93-7. 